\title{
Diagnosing symptomatic cancer in the NHS
}

\author{
Fast track referral is one part of an improving picture
}

\author{
William Hamilton professor of primary care diagnostics \\ College House, St Luke's Campus, Exeter EX1 2LU, UK
}

In a linked article, Møller and colleagues ${ }^{1}$ present a neat analysis of the use of the fast track referral pathway for patients with suspected cancer in 2009 (two week wait system) by English general practices and subsequent cancer mortality in patients from these practices. In 2009, this fast track pathway enabled access to a specialist opinion or diagnostic test within two weeks for eligible patients. Møller and colleagues studied three measures: the referral ratio (each practice's use of the two week wait system compared with other practices), detection rate (percentage of cancers in a practice that were detected via the two week clinics), and conversion rate (proportion of patients who went through the two week wait system and who were then shown to have cancer). Practices with a high referral ratio and those with a high detection rate had reduced cancer mortality, although the conversion rate showed no association.

Cancer survival in England has steadily improved since 1995, and this improvement is accelerating. ${ }^{2}$ Even so, we remain stubbornly behind other European countries, although the gap is narrowing for some cancers. ${ }^{2}$ This improvement is accompanied by a reduction in emergency presentations for cancer, from $25 \%$ overall in 2006 , to $20 \%$ by $2013 .{ }^{3}$ Times to diagnosis of cancer have also fallen, although largely for patients whose symptoms made them eligible for a two week wait appointment under the old guidelines from the National Institute for Health and Care Excellence (NICE) ${ }^{3-5}$

Furthermore, diagnostic activity for cancer has risen relentlessly in primary care, with a $29 \%$ increase between 2010 and $2013^{6}$ and now over a million two week wait appointments occurring annually. The conversion rate in these clinics is falling steadily, but is matched by an increased detection rate. This is

accompanied by rapid increases in imaging and endoscopy, to the extent that these services are struggling to meet demand. ${ }^{78}$

It is tempting to conclude from the new study that increased use of the urgent referral pathway leads to lower cancer mortality. However, some of the reported association will be due to case mix; given the relatively small numbers of new cancers annually in any practice, random variation will mean that in some years, a practice could have disproportionate numbers of so called "easy" patients to diagnose. ${ }^{9}$ These patients will take the two week referral route, obtain early diagnosis and treatment, avoid emergency presentation with its extra mortality, and do well. ${ }^{10}$
For colorectal cancer, the optimum time between first reporting of a symptom to primary care and eventual diagnosis is a month, ${ }^{11}$ which equates to referral within two weeks, followed by timely investigation. Only by repeating the study over multiple years can we identify the precise contribution of case mix to Møller and colleagues' results.

The alternative interpretation for the study's findings-which is favoured by the authors - is that some practices have a higher propensity to refer. Practices will use the two week pathway for some patients at low risk but not no risk, who other practices will choose not to refer. For this behaviour to explain the findings, sufficient numbers of these additional patients would have to harbour cancer, and their expedited diagnosis would have to be accompanied by improved mortality. The size of the improved mortality in the higher referring group $(4 \%, 95 \%$ confidence interval $1 \%$ to $6 \%$ ) is compatible with this.

However, the extra patients referred would be expected to lower the conversion rate, yet this rate was not associated with a difference in mortality. The explanation is more powerful for the low referring group of practices, who had $7 \%$ (confidence interval $5 \%$ to $8 \%$ ) worse mortality. If patients meeting the previous NICE guidance were not being referred by this group, and suffering as a result, then low referral ratios could be associated with an unchanged conversion rate. Practices now receive reports on several cancer metrics each year. Practices with a consistently low use of the two week system should consider why that is so.

Oddly, the precise explanation may not matter much: both interpretations suggest that early diagnosis of symptomatic cancer is beneficial, and that doing so via the two week system helps. This is fortunate, because the revised NICE guidance has liberalised access to these clinics, as well as expanding in-house primary care testing. ${ }^{12}$ The public supports this direction, opting for testing even if the risk of cancer was as low as $1 \% .{ }^{13}$ Most primary care clinicians also support the liberalisation of investigation enshrined in the new NICE guidance, which has partly reduced the gap between public expectation and what was on offer in the NHS. Much of this liberalisation has occurred already, prompted by awareness campaigns and multiple initiatives in primary care. Furthermore, the new recommendations explicitly support general practitioners' use 
of their clinical judgment, saving them from having to make the patient fit the guidelines. ${ }^{12}$

The recent improvements in cancer diagnosis are real, large, and welcome. A 5\% reduction in the proportion of emergency presentations is over 16000 fewer cancer emergencies annually, and this is only one component of the improvement. One major question remains, however-the cost. Cancer diagnostics are expensive, with perhaps $£ 1$ bn ( $€ 1.35 \mathrm{bn}$; $\$ 1.52 \mathrm{bn})$ spent annually. It has been difficult to quantify the survival benefit from expedited diagnosis of symptomatic cancer. In simple terms, we know what we are paying, but don't know what we are getting. At least now we know we are getting something for the investment: Møller and colleagues' study adds further evidence to the survival and emergency admission figures, and all point in the same direction. It is better to develop cancer in the United Kingdom now than it was 10 years ago-and improved diagnostic facilities are a part of the reason why. It may be even better in another 10 years.

Competing interests: I have read and understood the BMJ policy on declaration of interests and declare that I was the clinical lead on the recent NICE guidelines, NG12.

Provenance and peer review: Commissioned, not externally peer reviewed.
1 Møller $\mathrm{H}$, Gildea C, Meechan D, et al. Use of the English urgent referral pathway for suspected cancer and mortality in patients with cancer: cohort study. BMJ2015;351:h5102. 2 Walters S, Benitez-Majano S, Muller P, et al. Is England closing the international gap in cancer survival? Br J Cancer 2015;113:848-60.

3 Public Health England. Routes to diagnosis 2006-2013, preliminary results. 2015. www. ncin.org.uk/view?rid=3026

4 Neal RD, Din NU, Hamilton W, et al. Comparison of cancer diagnostic intervals before and after implementation of NICE guidelines: analysis of data from the UK General Practice Research Database. Br J Cancer 2014;110:584-92.

5 National Institute for Health and Care Excellence. Referral guidelines for suspected cancer. NICE, 2005.

6 Rubin G, Gildea C, Wild S, et al. Assessing the impact of an English national initiative for early cancer diagnosis in primary care. Br J Cancer 2015;112(s1):S57-S64.

72020 Delivery. Horizon scanning - an evaluation of imaging capacity across the NHS in England. 2015. https://www.cancerresearchuk.org/sites/default/files/horizon_scanning_final.pdf.

8 Health Services Management Centre. Scoping the future-an evaluation of evaluation of evaluation of endoscopy endoscopy capacity across the capacity across the across the NHS in England. 2015. https://www.cancerresearchuk.org/sites/default/files/scoping_the_ future - final.pdf.

9 Lyratzopoulos G, Wardle J, Rubin G. Rethinking diagnostic delay in cancer: how difficult is the diagnosis? BMJ 2014;349:g7400.

10 McPhail S, Elliss-Brookes L, Shelton J, et al. Emergency presentation of cancer and short-term mortality. Br J Cancer 2013;109:2027-34.

11 Tørring ML, Frydenberg M, Hamilton W, et al. Diagnostic interval and mortality in colorectal cancer: U-shaped association demonstrated for three different datasets. J Clin Epidemiol 2012:65:669-78.

12 National Institute for Health and Care Excellence. Suspected cancer: recognition and referral [NG12]. 2015. www.nice.org.uk/guidance/NG12.

13 Banks J, Hollinghurst S, Bigwood L, et al. Preferences for cancer investigation: a vignette-based study of primary-care attendees. Lancet Oncol 2014;15:232-40.

Cite this as: BMJ 2015;351:h5311

(c) BMJ Publishing Group Ltd 2015 